NBER WORKING PAPER SERIES

\title{
LEARNING TO LIVE IN A LIQUIDITY TRAP
}

\author{
Jasmina Arifovic \\ Stephanie Schmitt-Grohé \\ Martín Uribe \\ Working Paper 23725 \\ http://www.nber.org/papers/w23725
NATIONAL BUREAU OF ECONOMIC RESEARCH
1050 Massachusetts Avenue
Cambridge, MA 02138 \\ August 2017
}

We thank for comments seminar participants at the July 2017 Stony Brook Workshop on Theoretical and Experimental Macroeconomics. The views expressed herein are those of the authors and do not necessarily reflect the views of the National Bureau of Economic Research.

NBER working papers are circulated for discussion and comment purposes. They have not been peer-reviewed or been subject to the review by the NBER Board of Directors that accompanies official NBER publications.

(C) 2017 by Jasmina Arifovic, Stephanie Schmitt-Grohé, and Martín Uribe. All rights reserved. Short sections of text, not to exceed two paragraphs, may be quoted without explicit permission provided that full credit, including $(\odot$ notice, is given to the source. 
Learning to Live in a Liquidity Trap

Jasmina Arifovic, Stephanie Schmitt-Grohé, and Martín Uribe

NBER Working Paper No. 23725

August 2017

JEL No. E03,E52

\section{ABSTRACT}

The Taylor rule in combination with the zero lower bound on nominal rates has been shown to create an unintended liquidity-trap equilibrium. The relevance of this equilibrium has been challenged on the basis that it is not stable under least-square learning. In this paper, we show that the liquidity-trap equilibrium is stable under social learning. The learning mechanism we employ includes three realistic elements: mutation, crossover, and tournaments. We show that agents can learn to have pessimistic sentiments about the central bank's ability to generate price growth, giving rise to a stochastically stable environment characterized by deflation and stagnation.

Jasmina Arifovic

Simon Fraser University

1590 Tunstall Blvd

Bowen Island

BC V0N 1G2

arifovic@sfu.ca

Stephanie Schmitt-Grohé

Department of Economics

Columbia University

420 West 118th Street, MC 3308

New York, NY 10027

and NBER

stephanie.schmittgrohe@ columbia.edu
Martín Uribe

Department of Economics

Columbia University

International Affairs Building

New York, NY 10027

and NBER

martin.uribe@columbia.edu 


\section{Introduction}

The Taylor rule in combination with the zero lower bound on nominal interest rates has been shown to open the door to unintended equilibria in which the economy is stuck in a liquidity trap with zero nominal rates and inflation and output below target. In this equilibrium, rational agents wake up each period with a pessimistic economic outlook. Guided by this sentiment, their demand for goods and services is weak. The central bank tries to counteract the weakness in aggregate demand by lowering interest rates. However, the zero lower bound prevents the monetary authority from fully offsetting the public's lack of confidence. As a result, the economy ends up with depressed levels of aggregate activity and prices and zero nominal rates. The next period, agents continue to hold pessimistic sentiments, which are now validated by the past experience. Thus, by the same mechanism at play in the first period, the economy ends again in a slump in the second period. Continuing with this logic yields a stationary environment with all the characteristics of a liquidity trap. This type of dynamics are sometimes invoked to explain aspects of the prolonged periods of below-target inflation observed in Japan since the 1990s and in the Eurozone since the onset of the Great Contraction.

The relevance of the Taylor-rule induced self-fulfilling liquidity trap has been challenged on the basis that it is not least-square learnable. In this branch of the literature the assumption of rational expectations is replaced by the assumption that agents form expectations by running regressions of observations of key macroeconomic indicators, such as inflation and output, onto economic fundamentals such as the natural of interest, productivity, or monetary policy shocks. Agents are assumed to update the regression coefficients recursively incorporating the new data generated as time goes on. Thus, the previous-period regression coefficient is a relevant endogenous state of the economy. The central result of this literature is that under least-square learning the liquidity-trap equilibrium exists, but fails to be stable. Specifically, it has been shown, that a small deviation of the regression coefficient from the value consistent with rational expectations triggers expectations dynamics (i.e., a sequence of regression coefficients) that diverge from the liquidity-trap rational expectations equilibrium.

In this paper we study the stability of the liquidity-trap equilibrium under an alternative learning hypothesis. We assume that expectations evolve through social learning. Social learning includes three realistic elements: tournaments, mutation, and crossover, which are standard features in genetic algorithms. In the context of an economic environment, these three elements are meant to describe plausible ways in which individuals change their perceived law of motion of the economy through social interactions. Tournaments reward agents 
with superior forecasting abilities, by allowing them to pass their views to other agents. This channel captures, for example, the influence exerted by professional economists and forecasters with proven economic models. Mutation allows for agents to have arbitrary revisions of expectations or new ideas, guaranteeing a variety of views in the population across time. And crossover incorporates the role of imitation, by permitting agents to adopt parts of somebody else's model of the economy. We embed the social learning algorithm in a canonical new Keynesian model driven by natural-rate shocks. Monetary policy is described by an active Taylor-type interest-rate feedback rule that respects the zero lower bound on nominal rates.

Our main finding is that the liquidity-trap equilibrium is stable under social learning. We show that agents can learn to have pessimistic sentiments about the central bank's ability to generate price growth, giving rise to a stochastically stable environment characterized by deflation and stagnation. Specifically, we show that, starting in the liquidity-trap equilibrium, after a large perturbation of individual perceived laws of motion, through social learning the economy returns to a dynamic path that is stable around the liquidity trap. This result obtains for fairly large initial disturbances to the individual perceived laws of motion. For example, in one experiment we replace agents' perceived laws of motion with the one associated with the intended rational expectations equilibrium (an equilibrium in which the economy fluctuates around the target levels of output and inflation), and show that the social learning algorithm leads the economy back to the liquidity trap.

Key in producing stability of the liquidity trap under social learning is the role of tournaments. The fittest individuals in the economy are those whose current perceived laws of motion can best explain historical data. As a result, if the historical data contains observations generated by the liquidity trap equilibrium, individuals who, for any reason (including pure luck), have perceived laws of motion close to the one associate with the liquidity-trap equilibrium will prevail in passing their views to others, causing the economy to gravitate towards the liquidity trap.

This paper is related to macroeconomic studies of the liquidity trap under rational and boundedly rational expectations. Benhabib, Schmitt-Grohé, and Uribe (2001) show that the combination of a Taylor-type interest-rate feedback rule and the zero lower bound constraint on nominal interest rates gives rise to multiple rational expectations equilibria, including one in which the economy is perpetually in a liquidity trap. The stability of the rational expectations equilibrium in monetary economies with a Taylor rule under least-square learning has been studied using the stochastic approximation results introduced to macroeconomics by Marcet and Sargent (1989) and the E-stability principle introduced by Evans and Honkapohja (2001). Bullard and Mitra (2002) and Evans and McGough (2017) show that 
the minimum-state-variable rational expectations equilibrium in a linearized New Keynesian model with a passive Taylor rule, including a pure interest rate peg, is not E-stable under decreasing-gain least-square learning. Evans, Guse, and Honkapohja (2008) study a discrete time version of the liquidity-trap model of Benhabib, Schmitt-Grohé, and Uribe (2001) and show analytically that the liquidity-trap rational expectations equilibrium is not E-stable under decreasing gain steady state learning for small enough degrees of price stickiness. This paper is most closely related to Arifovic, Bullard, and Kostyshyna (2012) who show that in a new-Keynesian model like the one studied here the rational expectations equilibrium is stable under social learning when the Taylor rule is globally active or globally passive. The present study differs from this paper along three dimensions. First, the monetary policy specification in the present model respects the Taylor principle and the zero lower bound on nominal rates. This means that monetary policy is neither globally active nor globally passive. Second, Arifovic, Bullard, and Kostyshyna (2012) do not study pure interest-rate pegs. In the model studied here, by contrast, the interest rate is pegged at zero (forced by the zero lower bound) when the economy is in a liquidity trap. And third, as a consequence of the zero lower bound, the present economy features multiple minimum-state-variable rational expectations equilibria, including the intended equilibrium and the liquidity trap, whereas the Arifovic, Bullard, and Kostyshyna (2012) economy features always a unique minimumstate-variable rational expectations equilibrium. We view the present study as an extension of the stability results under social learning of Arifovic, Bullard, and Kostyshyna (2012) to the liquidity-trap model of Benhabib, Schmitt-Grohé, and Uribe (2001).

The remainder of the paper is in seven sections. Section 2 presents the model. Section 3 shows the existence of multiple minimum-state-variable rational expectations equilibria in an economy driven by natural-rate shocks. Section 4 describes the social learning algorithm and its calibration. Section 5 presents the main result of the paper, namely, the stability of the liquidity-trap equilibrium under social learning. Section 6 shows that the intended equilibrium is also stable under social learning. Section 7 presents a sensitivity analysis, and section 8 concludes.

\section{The Model}

The theoretical environment into which we embed social learning is the canonical newKeynesian model whose main components are expectations-augmented versions of the IS and Phillips curves. The linearized form of these two equations are, respectively,

$$
y_{t}=y_{t+1}^{e}-\sigma^{-1}\left(i_{t}-\pi_{t+1}^{e}\right)+\sigma^{-1} r_{t}^{n}
$$




$$
\pi_{t}=\kappa y_{t}+\beta \pi_{t+1}^{e},
$$

where $y_{t}$ denotes the output gap, $y_{t+1}^{e}$ denotes the period- $t$ expected value of $y_{t+1}, \pi_{t}$ denotes the deviation of the inflation rate from target, $\pi_{t+1}^{e}$ denotes the period- $t$ expected value of $\pi_{t+1}, i_{t}$ denotes the deviation of the nominal interest rate from its steady-state value, denoted $i^{*}, r_{t}^{n}$ is an exogenous and stochastic shock to the natural rate of interest taking on the values $r_{H}^{n}$ and $r_{L}^{n}<r_{H}^{n}$ with transition probability matrix

$$
\left[\begin{array}{cc}
\rho_{H} & 1-\rho_{H} \\
1-\rho_{L} & \rho_{L}
\end{array}\right]
$$

where $\rho_{i} \equiv \operatorname{Prob}\left(r_{t+1}^{n}=r_{i}^{n} \mid r_{t}^{n}=r_{i}^{n}\right) \in(0,1)$ for $i=H, L$, and $\sigma>0, \kappa>0$, and $\beta \in(0,1)$ are parameters.

Letting

$$
z_{t} \equiv\left[\begin{array}{c}
y_{t} \\
\pi_{t}
\end{array}\right]
$$

we can write the system (1)-(2) as

$$
z_{t}=a+b z_{t+1}^{e}+c i_{t}+d r_{t}^{n}
$$

where

$$
a \equiv\left[\begin{array}{l}
0 \\
0
\end{array}\right], b \equiv G^{-1}\left[\begin{array}{cc}
1 & \sigma^{-1} \\
0 & \beta
\end{array}\right], c=-d \equiv G^{-1}\left[\begin{array}{c}
-\sigma^{-1} \\
0
\end{array}\right] \text {, and } G \equiv\left[\begin{array}{cc}
1 & 0 \\
-\kappa & 1
\end{array}\right]
$$

As we will see shortly, this compact notation facilitates the characterization of model's dynamics.

The central bank sets the nominal interest rate according to a Taylor rule, and the interest rate is bounded below by zero,

$$
i_{t}=\max \left\{-i^{*}, \phi_{\pi} \pi_{t}+\phi_{y} y_{t}\right\}
$$

where $\phi_{\pi}>1$ and $\phi_{y}>0$ are policy parameters. To see that the specified interest-rate feedback rule satisfies the zero lower bound on nominal rates, note that because $i_{t}$ is the deviation of the nominal interest rate from its target $i^{*}$, the nominal interest rate itself equals $i_{t}+i^{*}$, and the zero lower bound constraint is $i_{t}+i^{*} \geq 0$. Following the compact notation introduced above, the interest-rate feedback rule can be written as

$$
i_{t}=\max \left\{-i^{*},\left[\begin{array}{ll}
\phi_{y} & \phi_{\pi}
\end{array}\right] z_{t}\right\}
$$




\section{Three Rational Expectations Equilibria}

In this section we characterize three minimum-state-variable rational expectations (MSV$\mathrm{RE}$ ) equilibria. In the first equilibrium the economy never falls into a liquidity trap (the intended rational expectations equilibrium), in the second the economy is always in the liquidity trap (the unintended rational expectations equilibrium), and in the third the economy occasionally falls into a liquidity trap. We derive conditions under which each of these three rational expectations equilibria exist. In principle, any combination of these equilibria can coexist, and there may also exist parameter specifications under which none of these equilibria exist. Thus, we will also explore the constellation of equilibria that emerges under different parameter configurations of interest.

\subsection{The No-Liquidity-Trap Rational Expectations Equilibrium}

Here we consider the existence of a rational expectations equilibrium in which the zero lower bound constraint on the nominal interest rate is never binding. This equilibrium is the one intended by the policymaker, because it is the one in which the inflation rate remains close to the intended target and the output gap fluctuates around zero. We refer to this equilibrium as the RE-NB equilibrium. The fact that we restrict attention to MSV-RE equilibria means that equilibrium variables must be functions of the natural rate of interest only. Since $r_{t}^{n}$ is assumed to follow a two-state Markov process, $z_{t}$ can take on only two values.

For an RE-NB equilibrium to exist, the nominal interest rate must be strictly positive at all times, or

$$
\Phi z^{n b}>-i^{*}
$$

where $z^{n b}$ is a $4 \times 1$ vector collecting the values of output and inflation in the states $r_{H}^{n}$ and $r_{L}^{n}$ as follows

$$
z^{n b}=\left[\begin{array}{c}
z_{H}^{n b} \\
z_{L}^{n b}
\end{array}\right] \text { with } z_{H}^{n b}=\left[\begin{array}{c}
y_{H}^{n b} \\
\pi_{H}^{n b}
\end{array}\right] \text { and } z_{L}^{n b}=\left[\begin{array}{c}
y_{L}^{n b} \\
\pi_{L}^{n b}
\end{array}\right]
$$

and $y_{H}^{n b}, y_{L}^{n b}$ and $\pi_{H}^{n b}, \pi_{L}^{n b}$ denote the equilibrium values of $y_{t}$ and $\pi_{t}$ when $r_{t}^{n}$ takes the values $r_{H}^{n}$ and $r_{L}^{n}$, respectively. The object $\Phi$ is a $2 \times 4$ matrix of policy parameters given by

$$
\Phi \equiv\left[\begin{array}{c}
\Phi_{H} \\
\Phi_{L}
\end{array}\right] ; \text { with } \Phi_{H}=\left[\begin{array}{llll}
\phi_{y} & \phi_{\pi} & 0 & 0
\end{array}\right] \text { and } \Phi_{L}=\left[\begin{array}{cccc}
0 & 0 & \phi_{y} & \phi_{\pi}
\end{array}\right] \text {. }
$$

Letting $z_{H}^{n b, e}$ denote the expected value of $z_{t+1}$ conditional on $z_{t}=z_{H}^{n b}$ and using a similar 
definition for $z_{L}^{n b, e}$, we have that under the RE-NB equilibrium

$$
z_{H}^{n b, e}=\rho_{H} z_{H}^{n b}+\left(1-\rho_{H}\right) z_{L}^{n b}
$$

and

$$
z_{L}^{n b, e}=\left(1-\rho_{L}\right) z_{H}^{n b}+\rho_{L} z_{L}^{n b} .
$$

Now letting

$$
\Gamma_{H} \equiv\left[\begin{array}{cccc}
\rho_{H} & 0 & 1-\rho_{H} & 0 \\
0 & \rho_{H} & 0 & 1-\rho_{H}
\end{array}\right] \text { and } \Gamma_{L} \equiv\left[\begin{array}{cccc}
1-\rho_{L} & 0 & \rho_{L} & 0 \\
0 & 1-\rho_{L} & 0 & \rho_{L}
\end{array}\right]
$$

we have that

$$
z_{H}^{n b, e}=\Gamma_{H} z^{n b}
$$

and

$$
z_{L}^{n b, e}=\Gamma_{L} z^{n b} .
$$

This allows us to write equation (3) in the states $H$ and $L$ as

$$
z_{H}^{n b}=a+b \Gamma_{H} z^{n b}+c \Phi_{H} z^{n b}+d r_{H}^{n}
$$

and

$$
z_{L}^{n b}=a+b \Gamma_{L} z^{n b}+c \Phi_{L} z^{n b}+d r_{L}^{n} .
$$

Collecting terms we have

$$
z^{n b}=A^{n b}+B^{n b} z^{n b}+D r^{n},
$$

where

$$
A^{n b} \equiv\left[\begin{array}{c}
a \\
a
\end{array}\right], B^{n b} \equiv\left[\begin{array}{c}
b \Gamma_{H}+c \Phi_{H} \\
b \Gamma_{L}+c \Phi_{L}
\end{array}\right], D \equiv\left[\begin{array}{cc}
d & \emptyset \\
\emptyset & d
\end{array}\right], \text { and } r^{n} \equiv\left[\begin{array}{c}
r_{H}^{n} \\
r_{L}^{n}
\end{array}\right] .
$$

Solving for $z^{n b}$ yields

$$
z^{n b}=\left(I-B^{n b}\right)^{-1}\left(A^{n b}+D r^{n}\right) .
$$

The rational expectations equilibrium with a never binding zero lower bound (RE-NB) exists if the value of $z^{n b}$ implied by this expression satisfies condition (5). 


\subsection{A Rational Expectations Equilibrium With A Perpetual Liq- uidity Trap}

In this equilibrium, which we denote $\mathrm{RE}-\mathrm{AB}$, the zero lower bound on the nominal interest rate is always binding, that is,

$$
i_{t}=-i^{*}
$$

for all $t$, which means that the nominal interest rate is always zero, and the economy is stuck in a liquidity trap regardless of the realization of the natural rate. This equilibrium can be said to be unintended, as inflation is below target and the output gap is negative on average.

Proceeding in a similar fashion as in solving the RE-NB equilibrium, let

$$
z^{a b}=\left[\begin{array}{c}
z_{H}^{a b} \\
z_{L}^{a b}
\end{array}\right], z_{H}^{a b}=\left[\begin{array}{c}
y_{H}^{a b} \\
\pi_{H}^{a b}
\end{array}\right] \text {, and } z_{L}^{a b}=\left[\begin{array}{c}
y_{L}^{a b} \\
\pi_{L}^{a b}
\end{array}\right]
$$

denote the values of $z_{t}$ in the RE-AB equilibrium when the natural rate takes the values $r_{H}^{n}$ or $r_{L}^{n}$, respectively.

Then using equation (3) we can write the dynamics associated with the RE-AB equilibrium as

$$
z_{H}^{a b}=a+b \Gamma_{H} z^{a b}-c i^{*}+d r_{H}^{n}
$$

and

$$
z_{L}^{a b}=a+b \Gamma_{L} z^{a b}-c i^{*}+d r_{L}^{n},
$$

where $\Gamma_{H} z^{a b}$ and $\Gamma_{L} z^{a b}$ denote the expected value of $z_{t+1}$ when $r_{t}^{n}=r_{H}^{n}$ and $r_{t}^{n}=r_{L}^{n}$, respectively. Combining these two laws of motion yields

$$
z^{a b}=A^{a b}+B^{a b} z^{a b}+D r^{n}
$$

where

$$
A^{a b} \equiv\left[\begin{array}{c}
a-c i^{*} \\
a-c i^{*}
\end{array}\right] \text { and } B^{a b} \equiv\left[\begin{array}{l}
b \Gamma_{H} \\
b \Gamma_{L}
\end{array}\right] .
$$

Solving for $z^{a b}$ we have

$$
z^{a b}=\left(I-B^{a b}\right)^{-1}\left(A^{a b}+D r^{n}\right) .
$$

The vector $z^{a b}$ is an equilibrium if it satisfies,

$$
\Phi z^{a b} \leq-i^{*}
$$




\subsection{A Rational Expectations Equilibrium With An Occasional Liquidity Trap}

We define a rational expectations equilibrium with an occasionally binding (RE-OB) zero lower bound as a MSV-RE equilibrium in which the economy enjoys high levels of aggregate activity and inflation near its intended target when the natural rate is high, but falls into a liquidity trap with zero interest rates, deflation, and depressed levels of aggregate activity when the natural rate is low.

Let the equilibrium value of the endogenous vector be denoted by $z_{L}^{o b}$, if $r_{t}^{n}=r_{L}^{n}$ and by $z_{H}^{o b}$ if $r_{t}^{n}=r_{H}^{n}$. Further, let

$$
z^{o b}=\left[\begin{array}{c}
z_{H}^{o b} \\
z_{L}^{o b}
\end{array}\right], z_{H}^{o b} \equiv\left[\begin{array}{c}
y_{H}^{o b} \\
\pi_{H}^{o b}
\end{array}\right] \text {, and } z_{L}^{o b} \equiv\left[\begin{array}{c}
y_{L}^{o b} \\
\pi_{L}^{o b}
\end{array}\right]
$$

The requirement that the zero lower bound bind in the low state but not bind in the high state implies that

$$
\Phi_{H} z^{o b}>-i^{*}
$$

and

$$
\Phi_{L} z^{o b} \leq-i^{*}
$$

The equilibrium conditions (3) and (4) can then be written as

$$
z_{H}^{o b}=a+b \Gamma_{H} z^{o b}+c \Phi_{H} z^{o b}+d r_{H}^{n}
$$

and

$$
z_{L}^{o b}=a+b \Gamma_{L} z^{o b}-c i^{*}+d r_{L}^{n}
$$

In turn we can write these two equations as

$$
z^{o b}=A^{o b}+B^{o b} z^{o b}+D r^{n}
$$

where

$$
A^{o b} \equiv\left[\begin{array}{c}
a \\
a-c i^{*}
\end{array}\right] \text { and } B^{o b} \equiv\left[\begin{array}{c}
b \Gamma_{H}+c \Phi_{H} \\
b \Gamma_{L}
\end{array}\right] .
$$

Solving for $z^{o b}$, we obtain

$$
z^{o b}=\left(I-B^{o b}\right)^{-1}\left(A^{o b}+D^{o b} r^{n}\right) .
$$


The vector $z^{o b}$ is a RE-OB equilibrium if

$$
\Phi_{H} z^{o b}>-i^{*} \text { and } \Phi_{L} z^{o b} \leq-i^{*}
$$

\subsection{Multiple MSV Rational Expectations Equilibria}

As mentioned earlier, in principle, any combination of the three MSV-RE equilibria we just characterized can coexist. To give an idea of what combinations of equilibria actually arise for plausible calibrations, we proceed to parameterizing the model. The analysis here builds on Benhabib, Schmitt-Grohé, and Uribe (2001) who show that in the absence of fundamental shocks the combination of an active monetary policy stance $\left(\phi_{\pi}>1\right)$ and the zero lower bound results in two MSV-RE equilibria, namely, the one in which the zero lower bound never binds (RE-NB) and the one in which the zero lower bound always binds (RE-AB). A question that we wish to answer before tackling the issue of learning is whether this result carries over to an economy buffeted by fundamental shocks, in this case natural rate shocks. Of particular interest, therefore, is to explore how the features of the driving process $r_{t}^{n}$ (e.g., its volatility and persistence) can alter the type and combinations of equilibria that may arise.

To this end, we resort to a parameterization of the new-Keynesian model that is standard in the related literature. Table 1 summarizes it. We assume that the time unit is one quarter. We set $\beta=0.99, \sigma=2, \kappa=0.02, \phi_{y}=0.125, \phi_{\pi}=1.5$, and $i^{*}=\beta^{-1}-1$. The values of $\beta$, $\sigma$, and $\kappa$ are taken from Eggertsson and Woodford (2003), and the parameters of the Taylor rule, $\phi_{y}$ and $\phi_{\pi}$, are standard in the literature. The calibration of $i^{*}$ implies that the inflation target is zero, as assumed in much of the new-Keynesian literature (e.g., Woodford, 2003). We adopt a symmetric specification of the natural rate process and set $r_{H}^{n}=-r_{L}^{n}=0.0093$ and $\rho_{H}=\rho_{L}=0.675$. These parameter values imply that $r_{t}^{n}$ has a standard deviation of 3.72 percent at an annual rate and a serial correlation of 0.35, as in Woodford (1999). For this calibration of the natural rate process, there exist two minimum state variable rational expectations equilibria, one in which the zero lower bound constraint is always binding (RE$\mathrm{AB})$ and one in which it is never binding (RE-NB). The numerical equilibrium values of the laws of motion of output and inflation are

$$
z^{a b}=\left[\begin{array}{c}
y_{H}^{a b} \\
\pi_{H}^{a b} \\
y_{L}^{a b} \\
\pi_{L}^{a b}
\end{array}\right]=\left[\begin{array}{r}
0.0022 \\
-0.0099 \\
-0.0123 \\
-0.0103
\end{array}\right] \text { and } z^{n b}=\left[\begin{array}{c}
y_{H}^{n b} \\
\pi_{H}^{n b} \\
y_{L}^{n b} \\
\pi_{L}^{n b}
\end{array}\right]=\left[\begin{array}{r}
0.0064 \\
0.0002 \\
-0.0064 \\
-0.0002
\end{array}\right]
$$


Table 1: Calibration

\begin{tabular}{ccl}
\hline \hline Symbol & Value & Description \\
\hline$\beta$ & & \multicolumn{1}{c}{ Structural Parameters } \\
$\sigma$ & 0.99 & Subjective discount factor \\
$\kappa$ & 2 & Reciprocal of Intertemporal elasticity of substitution \\
$i^{*}$ & 0.02 & Output coefficient of the Phillips curve \\
$\phi_{\pi}$ & 0.0101 & Nominal interest rate target $\left(=\beta^{-1}-1\right)$ \\
$\phi_{y}$ & 0.125 & Onflation coefficient of the Taylor rule \\
& & \\
\hline$r_{H}^{n}$ & 0.0093 & Deviation of natural rate from steady state in state $H$ \\
$r_{L}^{n}$ & -0.0093 & Deviation of natural rate from steady state in state $\left.L=-r_{H}^{n}\right)$ \\
$\rho_{H}$ & 0.675 & Prob $\left(r_{t+1}^{n}=r_{H}^{n} \mid r_{t}^{n}=r_{H}^{n}\right)$ \\
$\rho_{L}$ & 0.675 & Prob $\left(r_{t+1}^{n}=r_{L}^{n} \mid r_{t}^{n}=r_{L}^{n}\right)\left(=\rho_{H}\right)$ \\
\hline & & $\quad$ Social Learning Algorithm \\
\hline$T_{I H}$ & 100 & Length of initial history \\
$T$ & 1000 & Length of simulation period \\
$N$ & 300 & Number of Agents \\
$p c$ & 0.1 & Probability of crossover \\
$p m$ & 0.1 & Probability of mutation \\
\hline \hline
\end{tabular}

Notes. The time unit is one quarter. The matrices $\Sigma$ and $\Sigma_{m}$, whose calibration is not included in the body of the table, are assumed to be diagonal and equal to each other. Their diagonals are set to $\left[\begin{array}{llll}0.0123 & 0.0103 & 0.0123 & 0.0103\end{array}\right]^{\prime}$. 
These vectors indicate that in the intended equilibrium (the RE-NB equilibrium) deviations of output and inflation from their steady-state values are symmetric and small, $0.64 \%$ and $8(=0.0002 \times 40000)$ basis points per year, respectively. However, when the economy falls into a liquidity-trap equilibrium, the RE-AB equilibrium, the economy deviates permanently and significantly from the intended steady state. The average output gap is -0.5 percent compared to 0 percent in the intended equilibrium. Thus, in the liquidity trap the economy experiences a permanent output loss. Similarly, average inflation falls from zero in the intended equilibrium to a deflation of 4 percent per year in the liquidity trap.

How typical is this combination of equilibria around the assumed values for the parameters defining the natural rate process? Figure 1 displays the combinations of MSV-RE equilibria that arise as one varies the parameters $r_{H}^{n}\left(=-r_{L}^{n}\right)$ expressed in percent per year, governing the standard deviation of $r_{t}^{n}$, and $\rho_{H}\left(=\rho_{L}\right)$, governing the serial correlation of $r_{t}^{n}$. These two parameters fully define the natural rate process within the two-state symmetric family we are considering. Dots indicate that the never-binding and always-binding equilibria (RE-NB and RE-AB) coexist. It is clear from the figure that this case is by far the most prevalent one. The baseline calibration for the natural rate process is indicated with a solid circle. We conclude that the main result of Benhabib, Schmitt-Grohé, and Uribe (2001), namely that in the absence of fundamental uncertainty, the combination of a zero-lower-bound and an active interest-rate rule gives rise to two MSV-RE equilibria, the RE-NB and the RE-AB, extends to an economy subject to natural-rate shocks.

Figure 1 further shows that as the serial correlation of the natural rate increases ( $\rho_{H}=$ $\rho_{L} \rightarrow 1$ ) other combinations of MSV rational expectations equilibria can arise, including the never-binding equilibrium together with the occasionally-binding equilibrium and the combination of all of the three equilibria characterized in the previous subsections (i.e., the never-binding, the always-binding, and the occasionally-binding equilibria). There are also parameterizations for which no MSV-RE equilibrium exists. These cases take place in a relatively small area of the space $\left(r_{H}^{n}, \rho_{H}\right)$, in which natural-rate shocks are highly persistent. It is noteworthy that if the intended equilibrium (i.e., the never-binding equilibrium) exists it never does so in isolation, but is always accompanied by at least one other equilibrium in which the zero lower bound is visited with some probability. This is an indication that the liquidity trap is a generic feature of this economy.

The analysis conducted thus far serves as a platform for delving into the issue of whether a liquidity trap is learnable. We take up this issue next. 
Figure 1: Existence of Multiple MSV Rational Expectations Equilibria in the $\left(r_{H}^{n}, \rho_{H}\right)$ Space

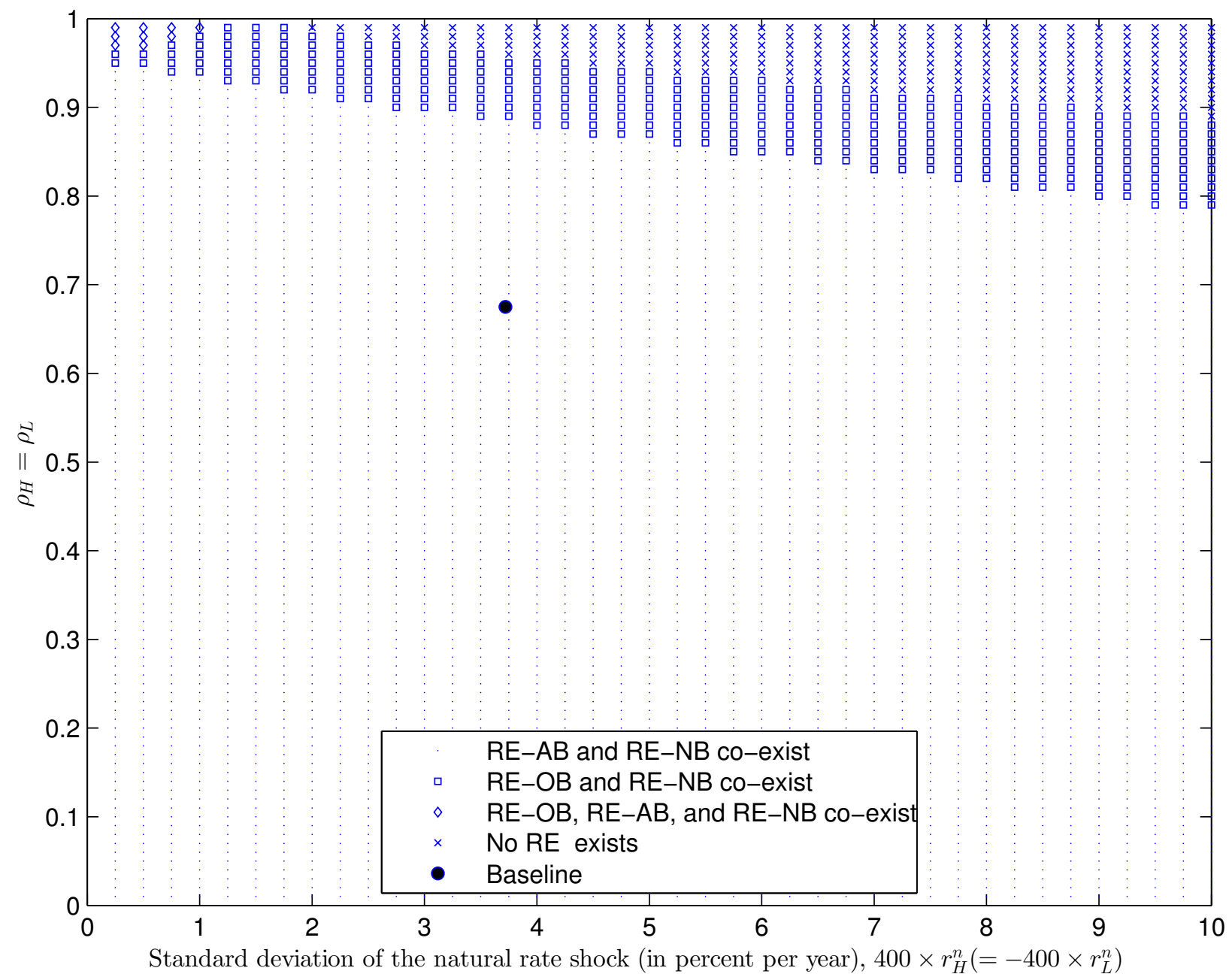

Notes. RE-AB, RE-NB, and RE-OB stand for MSV rational expectations equilibria in which the zero lower bound on nominal rates is, respectively, always binding, never binding, and occasionally binding. All parameters other than $r_{H}^{n}\left(=-r_{L}^{n}\right)$ and $\rho_{H}\left(=\rho_{L}\right)$ take the values shown in table 1. 


\section{The Social Learning Algorithm}

The learning algorithm follows Arifovic, Bullard, and Kostyshyna (2012). The economy is inhabited by a finite number of agents, each of which has a perceived law of motion (PLM) for output and inflation, which they use to form expectations about future values of these two variables. These laws of motion change over time due to social interaction and purely random changes in beliefs (mutations). There are two types of social interactions, one in which agents adopt parts of somebody else's PLM (crossover) and one in which agents adopt the beliefs of those who prove to be more successful in explaining observed outcomes (tournaments). The latter element of the social learning process, which can be conceived as the role played by professional economists and forecasters in the economy, is the one that contributes the most to the stability of the rational expectations equilibrium.

Suppose that there are $N$ individuals indexed by $i=1, \ldots, N$. Assume that individual $i$ has the perceived law of motion (PLM)

$$
z_{i H} \equiv\left[\begin{array}{c}
y_{i H} \\
\pi_{i H}
\end{array}\right] \text { and } z_{i L} \equiv\left[\begin{array}{c}
y_{i L} \\
\pi_{i L}
\end{array}\right]
$$

where $y_{i H}$ denotes the value of the output gap that agent $i$ believes obtains when the natural rate takes the value $r_{H}^{n}$. Similar definitions apply to $\pi_{i H}, y_{i L}$, and $\pi_{i L}$. Individual $i$ is assumed to know the current realization of the natural rate shock, $r^{n}$, and its transition probability matrix. Thus, individual $i$ forms expectations as follows

$$
z_{i H}^{e}=\rho_{H} z_{i H}+\left(1-\rho_{H}\right) z_{i L}
$$

and

$$
z_{i L}^{e}=\left(1-\rho_{L}\right) z_{i H}+\rho_{L} z_{i L},
$$

where $z_{i H}^{e}$ and $z_{i L}^{e}$ denote agent $i$ 's expectation of the value of $z$ in the next period conditional on the current value of $r^{n}$ being $r_{H}^{n}$ or $r_{L}^{n}$, respectively.

Aggregate conditional expectations, denoted $z_{H}^{e}$ and $z_{L}^{e}$, are defined as the cross-sectional averages of individual expectations, that is,

$$
z_{H}^{e}=\frac{1}{N} \sum_{i=1}^{N} z_{i H}^{e}
$$

and

$$
z_{L}^{e}=\frac{1}{N} \sum_{i=1}^{N} z_{i L}^{e}
$$


To obtain the actual law of motion (ALM), denoted $z_{H} \equiv\left[\begin{array}{ll}y_{H} & \pi_{H}\end{array}\right]^{\prime}$ and $z_{L} \equiv\left[\begin{array}{ll}y_{L} & \pi_{L}\end{array}\right]^{\prime}$, use (9) and (10) to eliminate $z_{H}^{e}$ and $z_{L}^{e}$ from equations (3) and (4), which yields

$$
z_{H}=a+b z_{H}^{e}+c i_{H}+d r_{H}^{n}
$$

and

$$
i_{H}=\max \left\{-i^{*},\left[\phi_{y} \phi_{\pi}\right] z_{H}\right\}
$$

if $r^{n}=r_{H}^{n}$, and

$$
z_{L}=a+b z_{L}^{e}+c i_{L}+d r_{L}^{n}
$$

and

$$
i_{L}=\max \left\{-i^{*},\left[\phi_{y} \phi_{\pi}\right] z_{L}\right\}
$$

if $r^{n}=r_{L}^{n}$.

Expressions (11) and (12) constitute a system of three equations in three unknowns, $y_{H}$, $\pi_{H}$, and $i_{H}$, given $z_{H}^{e}$ and $r_{H}^{n}$. Similarly, (13) and (14) are a system of three equations and three unknowns, $y_{L}, \pi_{L}$, and $i_{L}$, given $z_{L}^{e}$ and $r_{L}^{n}$. Each system can be solved separately each period. The way to solve each system is to first assume that the zero bound does not bind. If the solution satisfies the zero lower bound, then the equilibrium has been found. If the solution violates the zero lower bound, then set $i$ equal to $-i^{*}$ and solve again.

At this point, the social learning algorithm updates the individual PLMs by applying the three social improvements, crossover, mutation, and tournaments, in this order.

\subsection{Crossover}

All agents are randomly matched into pairs without replacement. Matched agents exchange each element of their PLMs with probability 0.5. That is, each matched pair flips a coin 4 times, once for each element of the PLM, exchanging the corresponding element of their PLMs if the toss results in, say, tail. More specifically, suppose agent $i_{1}$ is matched with agent $i_{2}$. Let the PLM of agent $i_{1}$ be the quadruple $\left\{y_{i_{1} H}, \pi_{i_{1} H}, y_{i_{1} L}, \pi_{i_{1} L}\right\}$, and the PLM of agent $i_{2}$ the quadruple $\left\{y_{i_{2} H}, \pi_{i_{2} H}, y_{i_{2} L}, \pi_{i_{2} L}\right\}$. Then, if, for example, the first and fourth coin tosses resulted in tails, elements 1 and 4 of their PLMs crossover, resulting in the following 
transformations of PLMs:

$$
\begin{aligned}
\text { old PLM of agent } i_{1} & =\left\{y_{i_{1} H}, \pi_{i_{1} H}, y_{i_{1} L}, \pi_{i_{1} L}\right\} \\
\text { new PLM of agent } i_{1} & =\left\{y_{i_{2} H}, \pi_{i_{1} H}, y_{i_{1} L}, \pi_{i_{2} L}\right\} \\
\text { old PLM of agent } i_{2} & =\left\{y_{i_{2} H}, \pi_{i_{2} H}, y_{i_{2} L}, \pi_{i_{2} L}\right\} \\
\text { new PLM of agent } i_{2} & =\left\{y_{i_{1} H}, \pi_{i_{2} H}, y_{i_{2} L}, \pi_{i_{1} L}\right\} .
\end{aligned}
$$

However, not all matched agents will engage in crossovers. With exogenous probability $1-p c$ matched agents walk away with their original PLMs. The parameter $p c$ denotes the probability that a matched pair of agents engage in crossover, so that each period about $p c \times 100$ percent of the matches engage in crossover and the remaining $(1-p c) \times 100$ percent retain their original PLMs.

\subsection{Mutation}

In the assumed social learning algorithm, mutation follows crossover. A mutation in the PLM of individual $i$ is modeled as follows. Let $z_{i} \equiv\left[y_{i H} \pi_{i H} y_{i L} \pi_{i L}\right]^{\prime}$ denote the PLM of individual $i$ prior to a mutation and let $z_{i}^{\prime}$ denote the PLM of individual $i$ after a mutation. The mutation follows a random walk process. Specifically,

$$
z_{i}^{\prime}=z_{i}+\Sigma_{m} \epsilon_{i}
$$

where $\epsilon_{i}$ is a $4 \times 1$ random vector with a standard normal distribution and $\Sigma_{m}$ is a $4 \times 4$ diagonal matrix with positive diagonal elements. The matrix $\Sigma_{m}$ is assumed to be the same for all agents $i$. We assume that not all individuals or all elements of an individual's PLM will be affected by a mutation in a given period. Specifically, we assume that with exogenous probability $\mathrm{pm}$ an element of an individual's PLM experiences a mutation. This probability is assumed to be independent across agents and across elements of the PLM.

\subsection{Tournament Selection}

The third mechanism by which the perceived law of motion in period $t$ is updated is tournament selection. The purpose of this element of the social learning algorithm is to promote the opinion (PLM) of agents with higher in-sample forecasting accuracy. The tournament selection process is based on a fitness measure for each individual PLM constructed as follows. In period $t$, individuals know the current and past realizations of all aggregate endogenous and exogenous variables, that is, they know $z_{k}$ and $r_{k}^{n}$ for $k=1, \ldots, t$. Suppose in period $t$ 
individual $i$ has the PLM $z_{i H}$ and $z_{i L}$. Based on this (period- $t$ ) perceived law of motion, in period $k$ individual $i$ would have forecasted output and inflation in period $k$ to be

$$
y_{i, k}^{f}= \begin{cases}\rho_{H} y_{i H}+\left(1-\rho_{H}\right) y_{i L} & \text { if } r_{k-1}^{n}=r_{H}^{n} \\ \left(1-\rho_{L}\right) y_{i H}+\rho_{L} y_{i L} & \text { if } r_{k-1}^{n}=r_{L}^{n}\end{cases}
$$

and

$$
\pi_{i, k}^{f}=\left\{\begin{array}{ll}
\rho_{H} \pi_{i H}+\left(1-\rho_{H}\right) \pi_{i L} & \text { if } r_{k-1}^{n}=r_{H}^{n} \\
\left(1-\rho_{L}\right) \pi_{i H}+\rho_{L} \pi_{i L} & \text { if } r_{k-1}^{n}=r_{L}^{n}
\end{array} .\right.
$$

Then the fitness of the period- $t$ PLM of agent $i$ for output and inflation is defined as

$$
F_{i, t}^{y}=-\frac{1}{t-1} \sum_{k=2}^{t}\left(y_{k}-y_{i, k}^{f}\right)^{2}
$$

and

$$
F_{i, t}^{\pi}=-\frac{1}{t-1} \sum_{k=2}^{t}\left(\pi_{k}-\pi_{i, k}^{f}\right)^{2}
$$

respectively. This fitness measure has the interpretation of the squared-sum of one-periodahead forecast errors. In each period, $N$ matches of two individuals are formed with replacement. (Recall that $N$ is the population size.) For each match the PLM for inflation and output, separately, that produced the better fitness measure is adopted. That is, the new beliefs that emerge from the social interaction of two individuals may have the PLM for inflation from one of them but the PLM for output from the other. This makes sense, because different economic agents might have different abilities at forecasting different variables.

\subsection{Calibration of the Social Learning Algorithm}

The calibration of the hyperparameters governing the social learning algorithm is as shown in the bottom panel of table 1 . We set $N$ denoting the number of individual agents to 300. This number ensures a significant variety of beliefs when we perturb the individual PLMs in the stability tests. To investigate the stability of the RE-AB equilibrium under social learning we assume that the economy has been operating under rational expectations for $T_{I H}$ quarters, the initial history, during which all agents share the same PLM, which coincides with the one associated with the RE-AB equilibrium. That is, during the initial history, $z_{i}=z^{a b}$ for all $i=1, \ldots, N$. We set $T_{I H}=100$ quarters. Immediately after the initial history, in period $T_{I H}+1$, individual PLMs are perturbed, and the simulation period with social learning begins. We set the simulation period, denoted $T$, equal to 1000 quarters. The perturbation of individual PLMs has mean zero and is independently distributed across 
agents. Specifically, in period $T_{I H}+1$, the perceived law of motion of individual $i$ is given by

$$
z_{i} \equiv\left[\begin{array}{c}
y_{i H} \\
\pi_{i H} \\
y_{i L} \\
\pi_{i L}
\end{array}\right]=z^{a b}+\Sigma \nu_{i}
$$

where $\nu_{i}$ denotes a vector of standard normal random variables independently distributed across time and agents. The diagonal matrix $\Sigma$ defines the standard deviation of the initial individual PLMs. We base the calibration of the diagonal of $\Sigma$ on the maximum absolute values of the output gap and inflation in the $\mathrm{RE}-\mathrm{AB}$ equilibrium, which are 0.0123 and 0.0103 , respectively (see equation (8)), that is, we set

$$
\Sigma=\left[\begin{array}{rrrr}
0.0123 & 0 & 0 & 0 \\
0 & 0.0103 & 0 & 0 \\
0 & 0 & 0.0123 & 0 \\
0 & 0 & 0 & 0.0103
\end{array}\right]
$$

We follow Arifovic, Bullard, and Kostyshyna (2012) and set the probability of crossover and mutation to 10 percent, $p m=p c=0.1$. Also following these authors, we assume that the standard deviations matrix, $\Sigma_{m}$, which governs the size of mutations, is equal to $\Sigma$. However, we assume that $\Sigma_{m}$ is constant, whereas Arifovic, Bullard, and Kostyshyna (2012) assume that its diagonal elements decrease over time. Our maintained assumption is more conservative, because mutation, while ensuring variety of beliefs over time, has also been found to be a destabilizing force in genetic learning algorithms.

\section{$5 \quad$ Stability of the Liquidity Trap Under Social Learning}

We are now ready to address the central question of this paper, namely, whether the liquidity trap, i.e., the $\mathrm{RE}-\mathrm{AB}$ equilibrium, is stable under social learning. As explained above, the way in which we gauge stability under social learning is to assume that all agents start with PLMs that are consistent with the liquidity-trap equilibrium (the RE-AB equilibrium). The economy runs under these PLMs for $T_{I H}$ quarters. In period $T_{I H}+1$ the random perturbation to the PLMs given in equation (15) takes place and the social learning algorithm begins. Convergence is assessed by checking in different ways whether through social learning the perceived laws of motion return to a neighborhood of their rational-expectations counterparts. Under social learning, by construction, PLMs never converge to a constant 
value, because of the randomness introduced by mutation. Thus, the criterion for stability is whether individual PLMs fluctuate around the rational-expectations PLM.

Figure 2 displays a simulated time path of the cross-sectional distribution of the perceived law of motions of output and inflation in states $H$ (top panels) and $L$ (bottom panels). The length of the simulation is $T_{I H}+T=1100$ quarters, and we drop the first $T_{I H}=100$ quarters. To facilitate the visualization of the transitional dynamics, the left column displays the initial 15 quarters after $T_{I H}$, and the right column displays the entire 1000 quarters after $T_{I H}$. Each panel shows the cross-sectional mean and a two-standard deviation band centered around the mean. The two-standard-deviation band of the initial cross-sectional distribution of PLMs is shown with crosses. As a point of reference, each panel also displays two horizontal lines indicating the law of motion associated with the liquidity-trap rational expectations equilibrium (the RE-AB equilibrium), the solid horizontal line, and the intended rational expectations equilibrium (the RE-NB equilibrium), the broken horizontal line.

Figure 2 shows that the PLMs fluctuate around the ones associated with the liquiditytrap rational-expectations equilibrium. By comparing the initial distribution of PLMs (the crosses) with the rest of the distribution, we see that the dispersion of beliefs shrinks quickly to its stationary level. These finding suggests that, at least in the particular simulation shown in the figure, the RE-AB equilibrium is stable under social learning.

Figure 2 provides information about the evolution of the perceived laws of motion for one particular simulation. Because both the social learning algorithm and the economy itself are subject to random shocks, the results will vary across simulations. To ascertain whether the pattern of stability under learning suggested by figure 2 holds more broadly, we consider next 1000 simulations of the economy under social learning, each of length $T_{I H}+T$ quarters. The results are shown in figure 3. Each dot corresponds to one simulation. The horizontal axis measures the cross sectional average absolute percent deviation of the individual PLMs from the liquidity-trap rational expectations PLM in period $T_{I H}+1$. For example, the horizontal axis in the top left panel measures $\frac{1}{N} \sum_{i=1}^{N} \frac{\left|y_{i H, T_{I H}+1}-y_{H}^{a b}\right|}{\left|y_{H}^{a b}\right|} \times 100$ and is denoted $\Delta_{1}$. The vertical axis displays the same measure in period $T_{I H}+T$ and is denoted $\Delta_{T}$. The other three panels are constructed in a similar fashion for the remaining elements of the PLM. As a reference, each panel displays the 45-degree line. The first thing to notice is that the initial average absolute disturbance of PLMs we impose is large in percentage terms, ranging from about 80 percent for $\pi_{H}$ to about 500 percent for $y_{H}$. The main result that emerges from the figure is that in each panel the cloud of points is well below the 45-degree line, which means that over the simulation period the economy returns to the liquidity-trap rational expectations law of motion. This result suggests that the liquidity trap is stable under social learning. 
Figure 2: Learning the Liquidity Trap: Evolution of the Cross-Sectional Distribution of PLMs
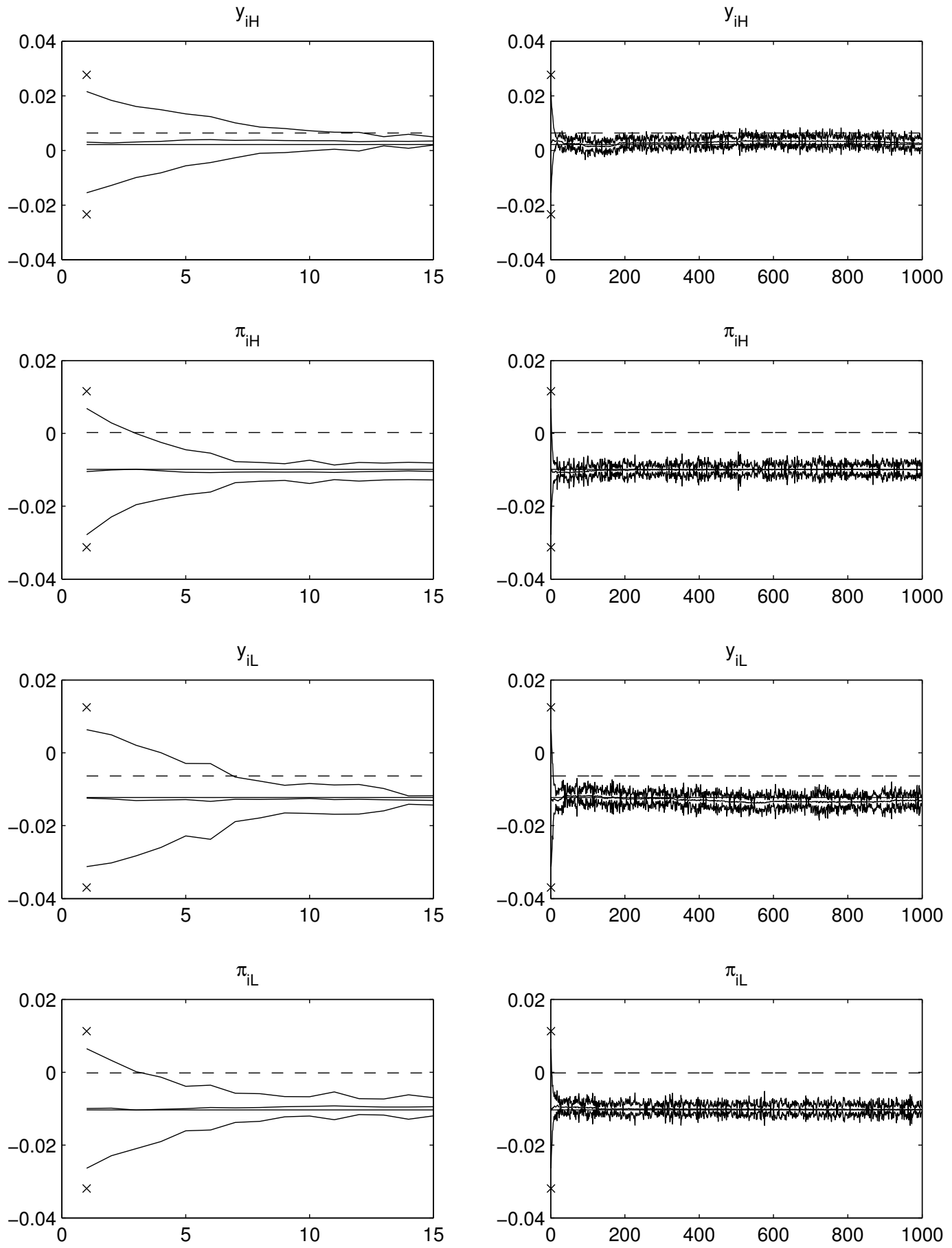

Notes. Both columns display the same objects, except that the first column displays only the first 15 periods of the simulation. Each panel in a given column shows the cross-sectional mean of individual PLMs and a two-standard deviation band around the mean. The solid horizontal line corresponds to the PLM associated with the liquidity-trap equilibrium (RE-AB equilibrium) and the broken horizontal line corresponds to the PLM associated with the intended equilibrium (RE-NB equilibrium). The two-standard-deviation band associated with the initial distribution of individual PLMs is shown with crosses. 
Figure 3: Percent Deviations of Perceived Laws of Motion from the Liquidity-Trap Equilibrium in Periods 1 and $T$
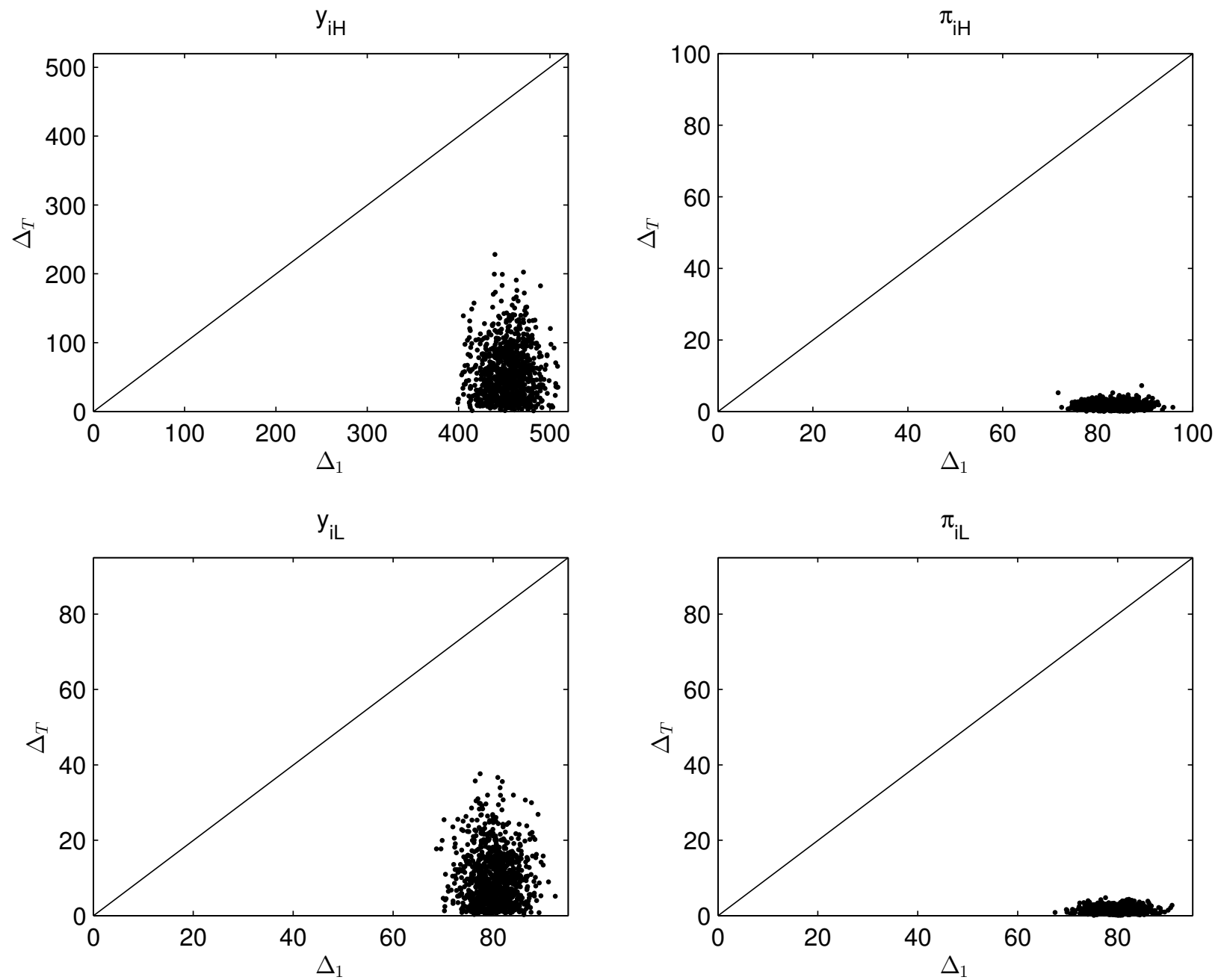

Note. $\Delta_{1}$ and $\Delta_{T}$ stand for the cross-sectional average percent absolute deviation of individual perceived laws of motion from the liquidity-trap rational expectations equilibrium in periods 1 and $T=1000$, respectively. Each dot corresponds to one simulation, and there are 1000 simulations. The length of each simulation is $T_{I H}+T$ quarters, and the first $T_{I H}$ quarters are dropped. The solid line corresponds to the 45-degree line. 


\section{Stability of the Intended Equilibrium Under Social Learning}

It is of use to compare the convergence of the economy to the liquidity-trap rational expectations equilibrium (the RE-AB equilibrium) with the convergence to the intended rational expectations equilibrium (the RE-NB equilibrium) to see if the transition dynamics differ in important ways. To this end, now assume that during the initial history (i.e., until period $\left.T_{I H}\right)$ the economy operates with all individual PLMs equal to the one associated with the intended rational expectation equilibrium, $z^{n b}$. In period $T_{I H}+1$ the individual PLMs suffer the same perturbation as the one applied in the analysis of the liquidity-trap equilibrium, that is, the distribution of PLMs in period $T_{I H}+1$ is given by

$$
z_{i} \equiv\left[\begin{array}{c}
y_{i H} \\
\pi_{i H} \\
y_{i L} \\
\pi_{i L}
\end{array}\right]=z^{n b}+\Sigma \nu_{i}=\left[\begin{array}{r}
0.0064 \\
0.0002 \\
-0.0064 \\
-0.0002
\end{array}\right]+\left[\begin{array}{rrrr}
0.0123 & 0 & 0 & 0 \\
0 & 0.0103 & 0 & 0 \\
0 & 0 & 0.0123 & 0 \\
0 & 0 & 0 & 0.0103
\end{array}\right] \nu_{i}
$$

All structural parameters and hyperparameters of the social learning algorithm take the values given in table 1. Figures 4 and 5 suggest that the intended equilibrium is stable under social learning. Importantly, the transitional dynamics of the learning process are qualitatively and quantitatively similar to those associated with the liquidity-trap equilibrium shown in figures 2 and 3. Notice that in figure 5 the percentage deviations from the intended rational expectations equilibrium appear much larger than in the liquidity-trap equilibrium. This is due to the fact that deviations are expressed in percent and that the level values of the endogenous variables (especially inflation) are smaller in the intended equilibrium than in the liquidity trap (see equation (8)). We conclude that the liquidity-trap equilibrium is not more difficult to learn than the intended rational expectations equilibrium.

To summarize, the main result of this section is that both the intended equilibrium (the RE-NB equilibrium) and the liquidity-trap equilibrium (the RE-AB equilibrium) are stable under social learning. The key source of stability is tournaments. This element of the learning algorithm allows agents with better forecasting ability to pass their perceived laws of motion to other agents. This is particularly important at the beginning of the transitional dynamics, because, since fitness is a backward-looking statistic, the fittest agents are those whose PLMs are closer to the rational expectations PLM in the beginning of the learning process (i.e., near period $T_{I H}+1$ ). In this regard, an important characteristic of the fitness measure is that it is a decreasing-gain average of squared forecast errors. Here the emphasis 
Figure 4: Learning the Intended Equilibrium: Evolution of the Cross-Sectional Distribution of PLMs
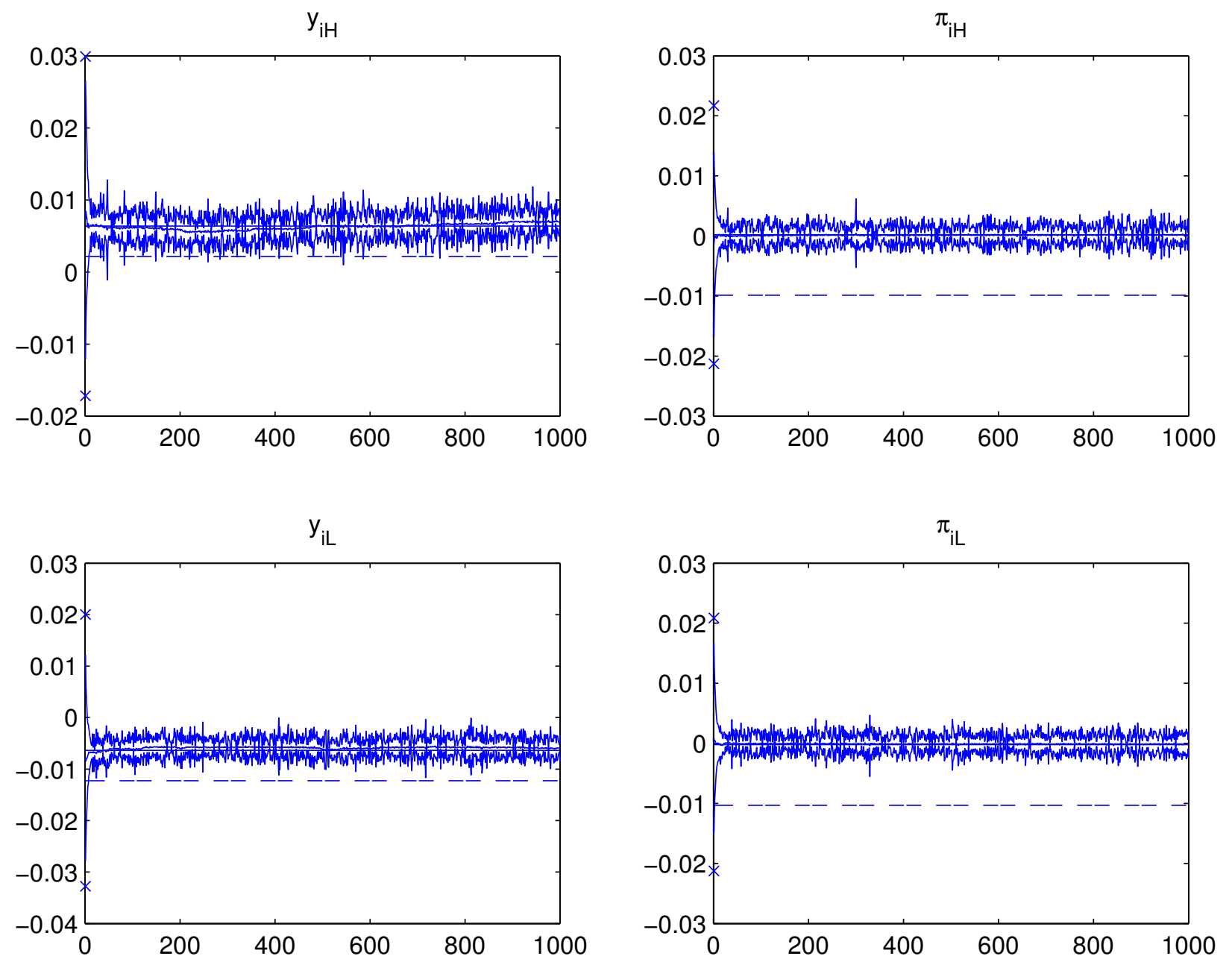

Notes. Each panel shows the cross-sectional mean of individual PLMs and a two-standard deviation band around the mean. The solid horizontal line corresponds to the PLM associated with the inteneded equilibrium (RE-NB) and the broken horizontal line corresponds to the PLM associated with the liquidity-trap equilibrium (RE-AB). The two-standard-deviation band associated with the initial distribution of individual PLMs is shown with crosses. 
Figure 5: Percent Deviations of Perceived Laws of Motion from the Intended Equilibrium in Periods 1 and $T$
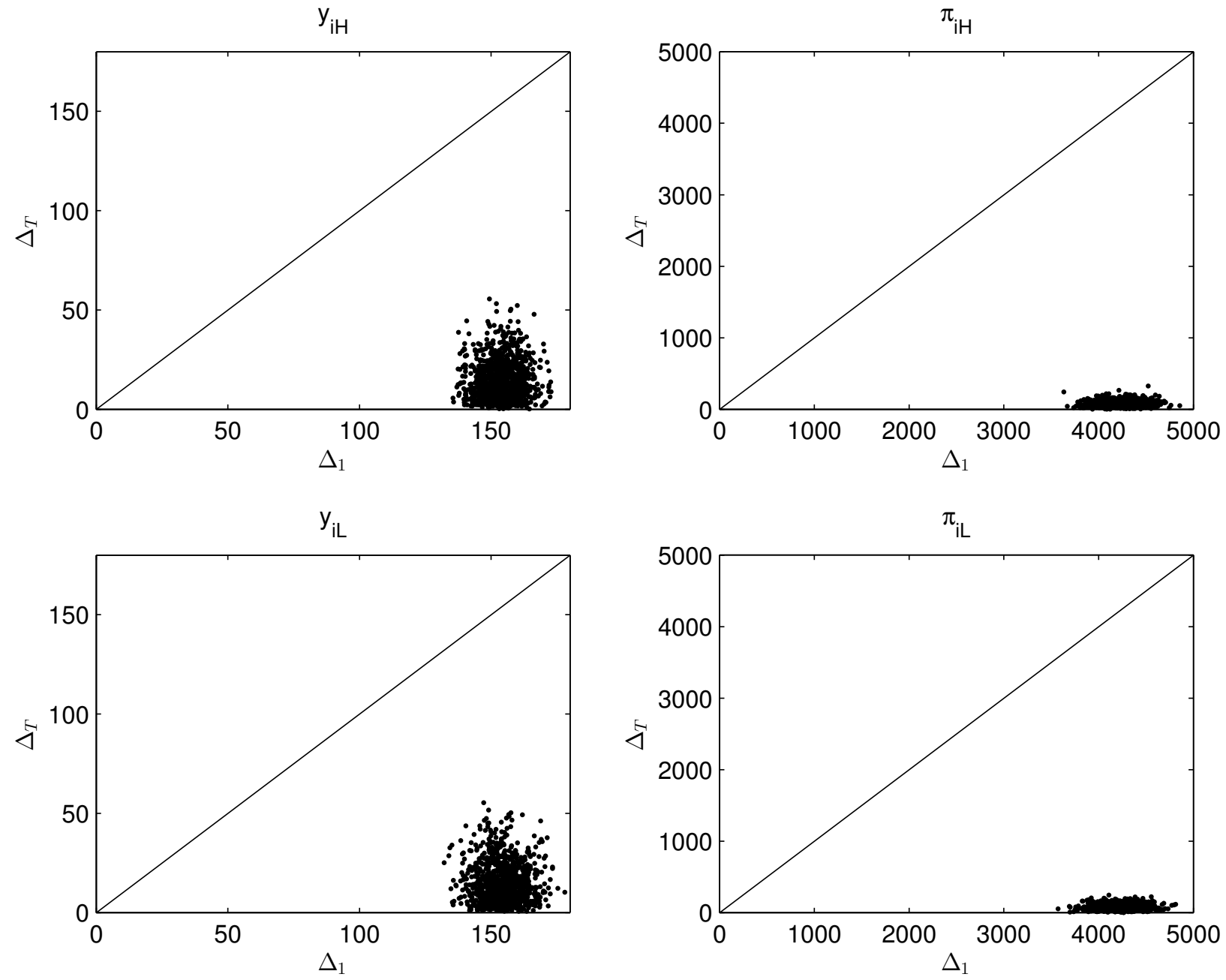

Note. $\Delta_{1}$ and $\Delta_{T}$ stand for the cross-sectional average percent absolute deviation of individual perceived laws of motion from the intended rational expectations equilibrium in periods 1 and $T=1000$, respectively. Each dot corresponds to one simulation, and there are 1000 simulations. The length of each simulation is $T_{I H}+T$ quarters, and the first $T_{I H}$ quarters are dropped. The solid line corresponds to the 45 -degree line. 
is on decreasing gain. This property can be seen from the fact that because all forecast errors are assigned the same weight, the most recent forecast error receives a smaller weight over time. We can show that this feature of tournaments is an important determinant of the learnability of the liquidity trap. Modeling fitness as an average of past forecasts with constant gain can make the liquidity trap non-learnable, at least for the relatively large dispersion of beliefs (embodied in the diagonal elements of the matrix $\Sigma$ ) considered in our numerical analysis. This result obtains for different specifications of constant gains, including geometric averaging and averaging over a fixed number of past periods.

\section{Sensitivity Analysis}

In this section we present two types of sensitivity tests. One consists in changing the various hyperparameters that define the social learning algorithm. The other consists in replacing the initial random disturbance in individual PLMs for one in which all agents are given the PLM corresponding to the intended rational expectations equilibrium.

\subsection{Varying the Hyperparameters of the Social Learning Algo- rithm}

Figure 6 presents a number of robustness checks of the stability of the liquidity trap under learning, with a focus on the hyperparameters of the social learning algorithm. This analysis is motivated by the fact that for these parameters there is little, if any, empirical information backing their calibration. Each row of the figure redoes figure 3 for a different value of one hyperparameter. All other structural and hyperparameters are set at the values shown in table 1. As a point of reference, the first row of the figure reproduces from figure 3 the predictions of the model under the baseline calibration.

The sensitivity test of greatest interest is the one that varies the probability of mutation, pm. The reason is that large values of this parameter can compromise the convergence of the learning algorithm, as discussed in, for example, Lux and Schornstein (2002) and Arifovic, Bullard, and Kostyshyna (2012). The baseline value of 0.1 is taken from the latter study. The second row of figure 6 displays the stability test for a value of $p m$ of 0.5 . As expected, the cloud of points moves closer to the 45-degree line, meaning that there is substantial

dispersion of beliefs even after 1000 quarters. This is in part a mechanical outcome, since exogenously 50 percent of the population is assumed to revise their beliefs in an arbitrary and random fashion every quarter. It is reassuring, however, that in spite of the high degree of mutation the equilibrium dynamics under learning continue to be stable, as reflected by 
Figure 6: Learning the Liquidity Trap: Sensitivity Analysis
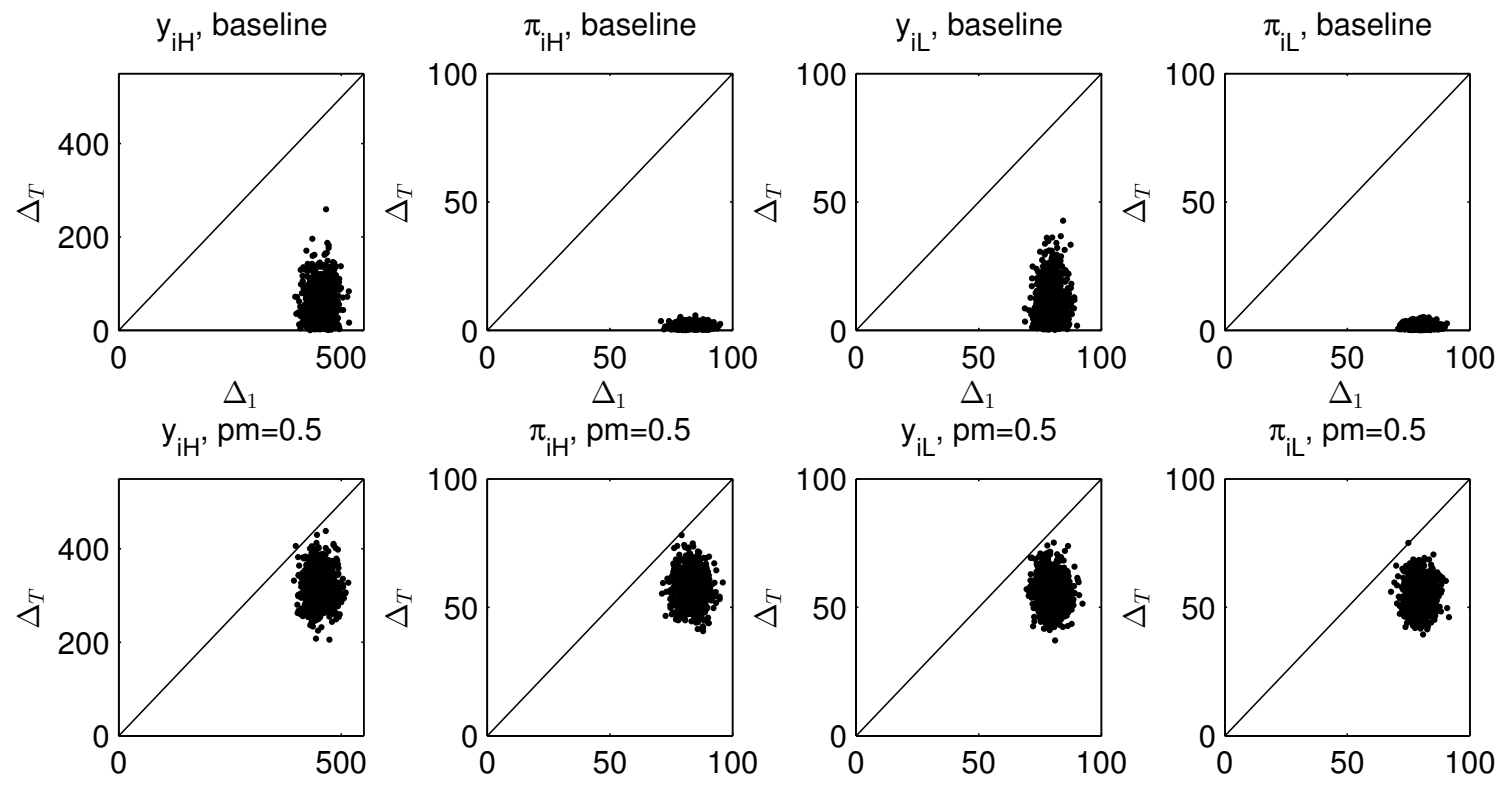

$\Delta_{1}$

$\Delta_{1}$

$\Delta_{1}$
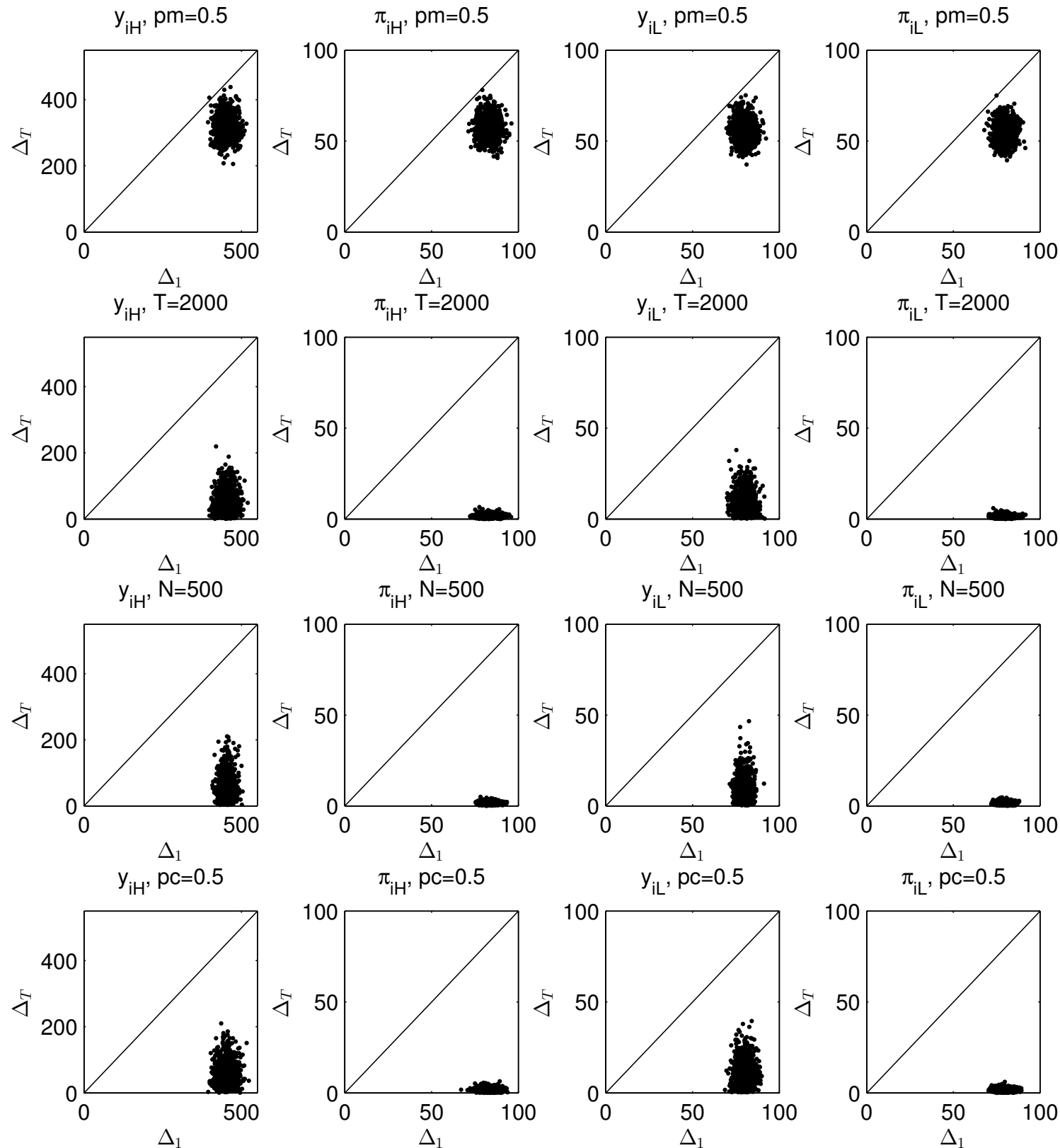

Note. All parameters other than the one indicated in each row are kept at the values shown in table 1 . See also note to figure 3 . 
the fact that the clouds of points for all elements of the perceived laws of motion remain below the 45-degree line.

Another sensitivity test of interest is to increase the length of the simulation period, because divergence can be slow to build up. The third row of figure 6 displays the stability test for $T=2000$ quarters, a simulation period twice as long as in the baseline test. Comparing the first and third rows of the figure reveals that the equilibrium dynamics remain stable after doubling the simulation period, suggesting absence of divergence. The last two sensitivity tests we perform are increasing the size of the population from 300 to 500 (row 4 of figure 6) and to increase the probability of crossover from 0.1 to 0.5 (row 5 of figure 6). Increasing the population size yields a richer variety of beliefs at every point in time, since mutation affects more agents. And an increase in the probability of crossover does not directly affect the aggregate perceived law of motion because the latter is an arithmetic mean of the individual PLMs. But it can affect the learning process indirectly by altering the fitness of individual PLMs. The stability of the liquidity trap under learning appears to be robust to both parameter changes.

\subsection{Convergence from the Intended Equilibrium to the Liquidity Trap}

Thus far, we have considered experiments in which the initial perturbation in the distribution of PLMs is mean preserving. Specifically, as described by equation (15), in period $T_{I H}+1$ the distribution of PLMs is centered around the one associated with the liquidity-trap rational expectations equilibrium, $z^{a b}$. We now test the stability of the liquidity trap under social learning by imposing a noncentered initial distribution of PLMs. To discipline the exercise, we pick a degenerate initial distribution in which all agents are assigned the PLM associated with the intended rational expectations equilibrium, $z^{n b}$.

The top panels of figure 7 display the transitional dynamics of the cross-sectional distribution of PLMs associated with this experiment for one simulation of 1000 quarters. The single cross that appears in period 1 in each plot indicates the corresponding element of $z^{n b}$. The figure shows that, as in the case of a mean-preserving initial PLM perturbation, the economy converges quickly to a stable distribution of PLMs around the liquidity-trap equilibrium. This finding is confirmed for 1000 simulations in the second row of panels, which shows the result of the convergence test described in section 5. By construction all 1000 points line up in a column, since all simulations start from the same degenerate crosssectional distribution of PLMs. The fact that the column of points lies below the 45-degree line implies that for all simulations the economy is closer to the liquidity trap at the end of 
Figure 7: Convergence from the Intended Equilibrium to the Liquidity Trap
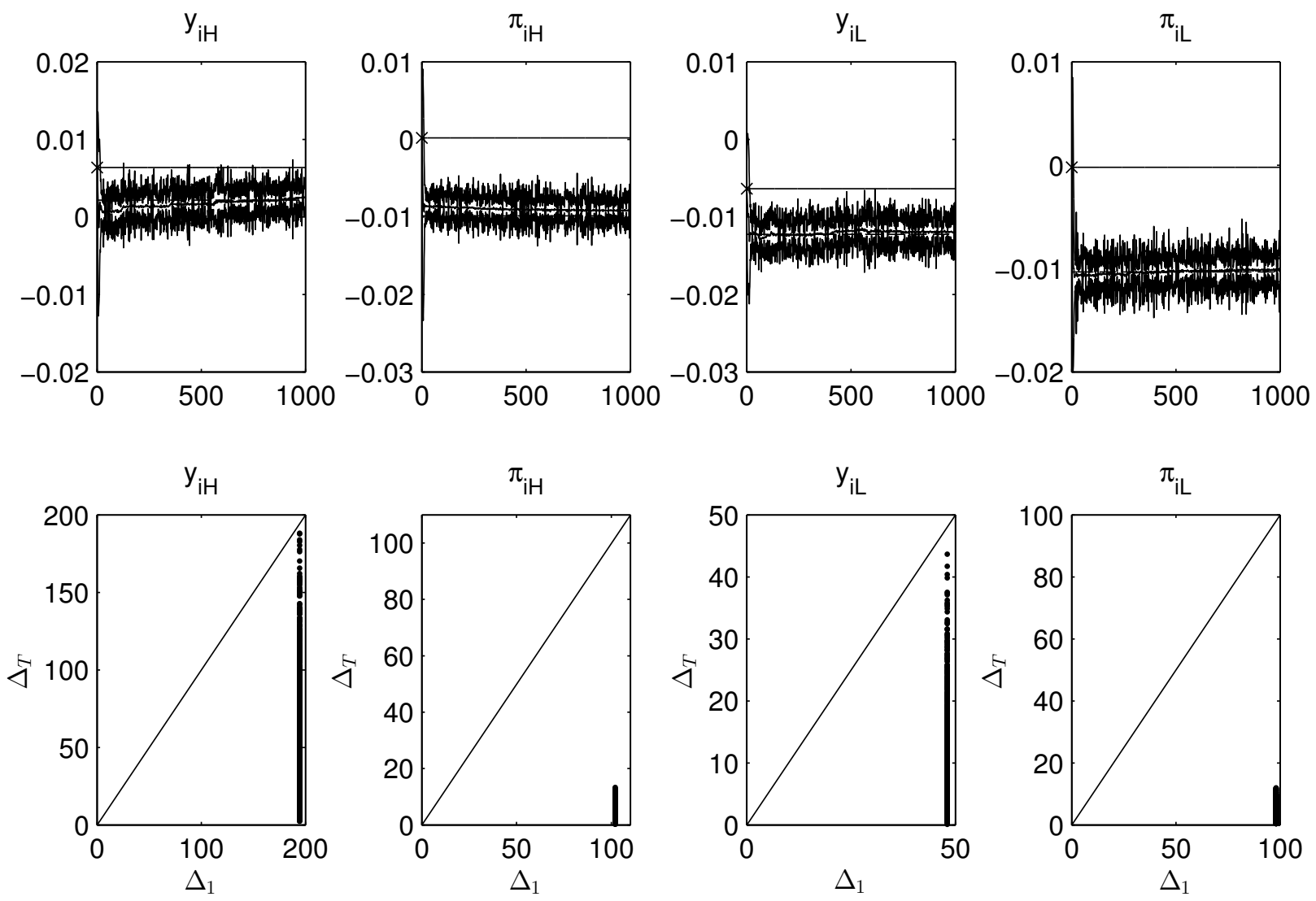

Note. See notes to figures 2 and 3 . 
the simulation period than it was at the beginning, suggesting stability of the liquidity trap equilibrium.

Why does the economy not stay at the intended equilibrium in spite of the fact that, as shown in section 6, this equilibrium is stable under social learning? The reason is that under social learning, history matters. And in the economy considered in this experiment, the history is one of an economy stuck in the liquidity trap. History matters in determining which members of the population will win tournaments, that is, convince other agents that they have the superior model of the economy. Immediately after the perturbation in period $T_{I H}+1$, all agents have the same PLM. However, the mutations that follow will, by chance, make some agents' PLMs closer to the one associated with the liquidity-trap equilibrium than others. Agents with PLMs closer to the liquidity-trap PLM will enjoy a better fit, since the historical data on which PLMs are evaluated stems from the liquidity-trap rational expectations equilibrium. Over time, these PLMs will spread across the population and dominate the beliefs in the economy.

\section{Conclusion}

The main strength of the Taylor principle is that, by promising to change interest rates by more than one-for-one with inflation, it ensures countercyclical real rates, which in turn, are conducive to aggregate stability. The main weakness of the Taylor principle is that it opens the door to unintended liquidity traps. Large enough downward revisions in inflationary expectations can lead the monetary authority, by the sheer active nature of the policy rule, to lower interest rates to zero, placing the economy in a liquidity trap, from which it might be difficult to escape.

The relevance of the Taylor-rule-induced liquidity trap has been put in doubt by a number of recent studies showing that it lacks stability when agents form expectations through leastsquare learning. This result can be placed in the context of theories that depart from the rational expectations hypothesis. There are multiple ways in which expectation formation can depart from rationality. The set of possibilities can be large even if one restricts attention to departures that converge to rationality. The contribution of this paper is to show that the liquidity-trap equilibrium is stable under social learning. The relevance of this result, lies in the view that social learning is no less appealing as a description of how agents form expectations in the real world than least square learning, especially given the fact that, as has been amply documented in the education and evolutionary sciences, learning has an important social component.

We believe that going forward a promising line of research is to devise ways to discipline 
the choice of expectations formation mechanisms. As in any model selection problem, the guiding principle should be to confront with actual data the predictions of models that incorporate different assumptions of how expectations are formed and to discriminate on the basis of goodness of fit. 


\section{References}

Arifovic, Jasmina, James Bullard, and Olena Kostyshyna, "Social Learning and Monetary Policy Rules," Economic Journal 123, March 2012, 38-76.

Benhabib, Jess, Stephanie Schmitt-Grohé, and Martín Uribe, "The Perils of Taylor Rules," Journal of Economic Theory 96, January-February 2001, 40-69.

Bullard, James, and Kaushik Mitra, "Learning about Monetary Policy Rules," Journal of Monetary Economics 49, 2002, 1105-1129.

Eggertsson, Gauti, and Michael Woodford, "The Zero Bound On Interest Rates And Optimal Monetary Policy," Brookings Papers On Economic Activity, 2003, 139-211.

Evans,George W., and Seppo Honkapohja, Learning and Expectations in Macroeconomics, Princeton, NJ: Princeton University Press, 2001.

Evans, George W., Eran Guse, and Seppo Honkapohja, "Liquidity Traps, Learning and Stagnation," European Economic Review 52, 2008, 1438-1463.

Evans, George W., and Bruce McGough, "Interest Rate Pegs in New Keynesian Models," Journal of Money Credit and Banking, March 31, 2017, forthcoming.

Lux, Thomas, and Sascha Schornstein, "Genetic Learning as an Explanation of Stylized Facts of Foreign Exchange Markets," Journal of Mathematical Economics 41, February 2005, 169-196.

Marcet, Albert, and Thomas Sargent, "Convergence of Least Squares Learning Mechanisms in Self-Referential Linear Stochastic Models," Journal of Economic Theory 48, August 1989, 337-368.

Woodford, Michael, "Optimal Monetary Policy Inertia," NBER Working Paper 7261, July 1999.

Woodford, Michael, Interest and Prices: Foundations of a Theory of Monetary Policy, Princeton, NJ: Princeton University Press, 2003. 